\title{
Some Results on Generalized Degree Distance
}

\author{
Asma Hamzeh, Ali Iranmanesh", Samaneh Hossein-Zadeh \\ Department of Mathematics, Faculty of Mathematical Sciences, Tarbiat Modares University, Tehran, Iran \\ Email: *iranmanesh@modares.ac.ir
}

Received January 14, 2013; revised February 15, 2013; accepted May 14, 2013

Copyright (C) 2013 Asma Hamzeh et al. This is an open access article distributed under the Creative Commons Attribution License, which permits unrestricted use, distribution, and reproduction in any medium, provided the original work is properly cited.

\begin{abstract}
In [1], Hamzeh, Iranmanesh Hossein-Zadeh and M. V. Diudea recently introduced the generalized degree distance of graphs. In this paper, we present explicit formulas for this new graph invariant of the Cartesian product, composition, join, disjunction and symmetric difference of graphs and introduce generalized and modified generalized degree distance polynomials of graphs, such that their first derivatives at $x=1$ are respectively, equal to the generalized degree distance and the modified generalized degree distance. These polynomials are related to Wiener-type invariant polynomial of graphs.
\end{abstract}

Keywords: Generalized Degree Distance; Cartesian Product; Join; Symmetric Difference; Composition; Disjunction

\section{Introduction}

A graph invariant is any function on a graph that does not depend on a labeling of its vertices. Topological indices and graph invariants based on the distances between the vertices of a graph are widely used in theoretical chemistry to establish relations between the structures and the properties of molecules. Topological indices provide correlations with physical, chemical and thermodynamic parameters of chemical compounds [2]. In this paper, we only consider simple and connected graphs. Let $G$ be a graph on $n$ vertices and $m$ edges. We denote the vertex and the edge set of $G$ by $V(G)$ and $E(G)$, respectively. As usual, the distance between the vertices $u$ and $v$ of $G$, denoted by $d_{G}(u, v)(d(u, v)$ for short), is defined as the length of a minimum path connecting them. We let $d_{G}(v)$ be the degree of a vertex $v$ in $G$. The eccentricity, denoted by $\varepsilon(v)$, is defined as the maximum distance from vertex $v$ to any other vertex. The diameter of a graph $G$, denoted by $\operatorname{diam}(G)$, is the maximum eccentricity over all vertices in a graph $G$.

The Cartesian product $G \times H$ of graphs $G$ and $H$ is a graph such that $V(G \times H)=V(G) \times V(H)$, and any two vertices $(a, b)$ and $(u, v)$ are adjacent in $G \times H$ if and only if either $a=u$ and $b$ is adjacent to $v$, or $b=v$ and $a$ is adjacent to $u$, see [3] for details. Let $G_{1}$ and $G_{2}$ be two graphs with disjoint vertex sets $V_{1}$ and $V_{2}$ and edge sets $E_{1}$ and $E_{2}$. The join $G_{1}+G_{2}$ is the graph union $G_{1} \cup G_{2}$ together with all the edges joining $V_{1}$ and $V_{2}$. The composition $G_{1}\left[G_{2}\right]$ is the

"Corresponding author. graph with vertex set $V_{1} \times V_{2}$ and $u=\left(u_{1}, v_{1}\right)$ is adjacent to $v=\left(u_{2}, v_{2}\right)$ whenever $\left(u_{1}\right.$ is adjacent with $\left.u_{2}\right)$ or $\left(u_{1}=u_{2}\right.$ and $v_{1}$ is adjacent to $\left.v_{2}\right),[3$, p. 185]. The disjunction $G \vee H$ of graphs $G$ and $H$ is the graph with vertex set $V(G) \times V(H)$ and $\left(u_{1}, v_{1}\right)$ is adjacent to $\left(u_{2}, v_{2}\right)$ whenever $u_{1} u_{2} \in E(G)$ or $v_{1} v_{2} \in E(H)$. The symmetric difference $G \oplus H$ of two graphs $G$ and $H$ is the graph with vertex set $V(G) \times V(H)$ and

$$
\begin{aligned}
E(G \oplus H)=\{ & \left(u_{1}, u_{2}\right)\left(v_{1}, v_{2}\right) \mid u_{1} v_{1} \in E(G) \text { or } \\
& \left.u_{2} v_{2} \in E(H) \text { but not both }\right\} .
\end{aligned} .
$$

The first Zagreb index was originally defined as $M_{1}(G)=\sum_{u \in V(G)} d_{G}(u)^{2}$ [4]. The first Zagreb index can be also expressed as a sum over edges of $G$, $M_{1}(G)=\sum_{u v \in E(G)}\left[d_{G}(u)+d_{G}(v)\right]$. We refer readers to [5] for the proof of this fact and for more information on Zagreb index. The first Zagreb coindex of a graph $G$ is defined in [6] as:

$$
\bar{M}_{1}(G)=\sum_{u v \notin E(G)}\left[d_{G}(u)+d_{G}(v)\right] .
$$

Let $d(G, k)$ be the number of pairs of vertices of a graph $G$ that are at distance $k, \lambda$ be a real number, and $W_{\lambda}(G)=\sum_{k \geq 1} d(G, k) k^{\lambda}$, that is called the Wienertype invariant of $G$ associated to $\lambda$, see $[7,8]$ for details. Additively weighted Harary index is defined in [9] as 


$$
H_{A}(G)=\sum_{\{u, v\} \subseteq V(G)} d^{-1}(u, v)\left(d_{G}(u)+d_{G}(v)\right) .
$$

Dobrynin and Kochetova in [10] and Gutman in [11] introduced a new graph invariant with the name degree distance that is defined as follows:

$$
D^{\prime}(G)=\sum_{\{u, v\} \subseteq V(G)} d(u, v)\left(d_{G}(u)+d_{G}(v)\right) .
$$

In [12], the modified degree distance was defined as follows:

$$
S^{*}(G)=\sum_{\{u, v\} \subseteq V(G)} d_{G}(u, v)\left(d_{G}(u) d_{G}(v)\right) .
$$

The generalized degree distance, denoted by $H_{\lambda}(G)$, is defined as follows in [1].

For every vertex $x$ and real number $\lambda, H_{\lambda}(x)$ is defined by $H_{\lambda}(x)=D^{\lambda}(x) d_{G}(x)$, where

$$
\begin{aligned}
D^{\lambda}(x)=\sum_{y \in V(G)} d^{\lambda}(x, y) . \text { We then define } \\
H_{\lambda}(G)=\sum_{x \in V(G)} H_{\lambda}(x)=\sum_{x \in V(G)} D^{\lambda}(x) d_{G}(x) \\
=\sum_{\{u, v\} \subseteq V(G)} d^{\lambda}(u, v)\left(d_{G}(u)+d_{G}(v)\right) .
\end{aligned}
$$

If $\lambda=0$, then $H_{\lambda}(G)=4 \mathrm{~m}$. When $\lambda=1$, this new topological index $\left(H_{\lambda}(G)\right)$ is equal to the degree distance (or Schultz index). There are many papers for studying this topological index, for example see [13-16]. Also if $\lambda=-1$, then $H_{\lambda}(G)=H_{A}(G)$. Therefore the study of this new topological index is important and we try to obtain some new results related to this topological index. The modified generalized degree distance, denoted by $H_{\lambda}^{*}(G)$, is defined in [1] as:

$$
H_{\lambda}^{*}(G)=\sum_{\{u, v\} \subseteq V(G)} d^{\lambda}(u, v)\left(d_{G}(u) d_{G}(v)\right) .
$$

If $\lambda=1$, then $H_{\lambda}^{*}(G)=S^{*}(G)$.

We construct graph polynomials having the property such that their first derivatives at $x=1$ are equal to the generalized degree distance, the modified generalized degree distance and Wiener-type invariant respectively. These polynomials are defined as follows:

$$
\begin{aligned}
& H_{\lambda}(G, x)=\sum_{\{u, v\} \leq V(G)}\left(d_{G}(u)+d_{G}(v)\right) x^{d^{\lambda}(u, v)}, \\
& H_{\lambda}^{*}(G, x)=\sum_{\{u, v\} \leq V(G)}\left(d_{G}(u) d_{G}(v)\right) x^{d^{\lambda}(u, v)},
\end{aligned}
$$

and

$$
W_{\lambda}(G, x)=\sum_{\{u, v\} \leq V(G)} x^{d^{\lambda}(u, v)} .
$$

The Wiener index of the Cartesian product of graphs was studied in [17,18]. In [19], Klavžar, Rajapakse and Gutman computed the Szeged index of the Cartesian product of graphs. In [9,20-24], exact formulae for the hyper-Wiener, the first Zagreb index, the second Zagreb index and Schultz polynomials of some graph operations were computed.

Throughout this paper, $C_{n}, P_{n}, K_{n}$ and $S_{n}$ denote the cycle, path, complete graph and star on $n$ vertices. The complement of a graph $G$ is a graph $H$ on the same vertices such that two vertices of $H$ are adjacent if and only if they are not adjacent in $G$. The graph $H$ is usually denoted by $\bar{G}$. Our other notations are standard and taken mainly from $[2,25,26]$.

In this paper we present explicit formulas for $H_{\lambda}(G)$ of graph operations containing the Cartesian product, composition, join, disjunction and symmetric difference of graphs and introduce generalized and modified generalized degree distance polynomials of graphs, such that their first derivatives at $x=1$ are respectively, equal to the generalized degree distance and the modified generalized degree distance. These polynomials are related with Wiener-type invariant polynomial of graphs.

\section{Main Results}

The aim of this section is to compute the generalized degree distance for five graph operations. We start with a lemma which gives some information about the number of vertices and edges of operations on two arbitrary graphs. For a given graph $G_{i}$, the number of vertices and edges will be denoted by $n_{i}$ and $m_{i}$, respectively.

Lemma 2.1. [3,20] Let $G$ and $H$ be graphs. Then we have:

a)

$$
\begin{aligned}
|V(G \times H)| & =|V(G \vee H)|=|V(G[H])|=|V(G \oplus H)| \\
& =|V(G)| \cdot|V(H)|, \\
|E(G \times H)| & =|E(G)| \cdot|V(H)|+|V(G)| \cdot|E(H)|, \\
|E(G+H)| & =|E(G)|+|E(H)|+|V(G)| \cdot|V(H)|, \\
|E(G[H])| & =|E(G)| \cdot|V(H)|^{2}+|E(H)| \cdot|V(G)|, \\
|E(G \vee H)| & =|E(G)| \cdot|V(H)|^{2}+|E(H)| \cdot|V(G)|^{2} \\
& -2|E(G)| \cdot|E(H)|,
\end{aligned}
$$

and

$$
\begin{aligned}
|E(G \oplus H)| & =|E(G)| \cdot|V(H)|^{2}+|E(H)| \cdot|V(G)|^{2} \\
& -4|E(G)| \cdot|E(H)| .
\end{aligned}
$$

b) The graph $G \times H$ is connected if and only if $G$ and $H$ are connected.

c) If $(a, c)$ and $(b, d)$ are vertices of $G \times H$, then $d_{G \times H}((a, c),(b, d))=d_{G}(a, b)+d_{H}(c, d)$.

d) The Cartesian product, join, composition, disjunction and symmetric difference of graphs are associative 
and all of them are commutative except the composition of graphs.

e)

$$
\begin{aligned}
& d_{G+H}(u, v) \\
& =\left\{\begin{array}{l}
0, u=v \\
1, u v \in E(G) \text { or } u v \in E(H) \text { or }(u \in V(G) v \in V(H)) . \\
2, \text { otherwise }
\end{array}\right.
\end{aligned}
$$

f)

$$
d_{G[H]}((a, b),(c, d))=\left\{\begin{array}{ll}
d_{G}(a, c), a \neq c \\
0, & a=c \& b=d \\
1, & a=c \& b d \in E(H) \\
2, & a=c \& b d \notin E(H)
\end{array} .\right.
$$

g)

$$
d_{G \vee H}((a, b),(c, d))=\left\{\begin{array}{l}
0, a=c \& b=d \\
1, a c \in E(G) \text { or } b d \in E(H) . \\
2, \text { otherwise }
\end{array}\right.
$$

h)

$$
\begin{aligned}
& d_{G \oplus H}((a, b),(c, d)) \\
& =\left\{\begin{array}{l}
0, a=c \& b=d \\
1, a c \in E(G) \text { or } b d \in E(H) \text { but not both. } \\
\text { 2, otherwise }
\end{array}\right.
\end{aligned}
$$

i) $d_{G \times H}((a, b))=d_{G}(a)+d_{H}(b)$.

j) $d_{G[H]}((a, b))=|V(H)| d_{G}(a)+d_{H}(b)$.

k) $d_{G+H}(a)=\left\{\begin{array}{l}d_{G}(a)+|V(H)|, a \in V G \\ d_{H}(a)+|V(G)|, a \in V(H)\end{array}\right.$.

1)

$$
\begin{aligned}
d_{G \vee H}((a, b)) & =|V(H)| d_{G}(a)+|V(G)| d_{H}(b) \\
& -d_{G}(a) d_{H}(b) .
\end{aligned}
$$

$\mathrm{m})$

$$
\begin{aligned}
d_{G \oplus H}((a, b))= & |V(H)| d_{G}(a)+|V(G)| d_{H}(b) \\
& -2 d_{G}(a) d_{H}(b) .
\end{aligned}
$$

In Theorem 2.2, we give a formula for the generalized degree distance of the join of two graphs.

Theorem 2.2. Let $G_{1}$ and $G_{2}$ be two graphs. Then

$$
\begin{aligned}
& H_{\lambda}\left(G_{1}+G_{2}\right) \\
= & n_{1} n_{2}\left(n_{1}+n_{2}\right)+4 n_{2} m_{1}+4 n_{1} m_{2}+M_{1}\left(G_{1}\right)+M_{1}\left(G_{2}\right) \\
& +2^{\lambda}\left(\bar{M}_{1}\left(G_{1}\right)+\bar{M}_{1}\left(G_{2}\right)\right)+2^{\lambda+1}\left(n_{2} \bar{m}_{1}+n_{1} \bar{m}_{2}\right) .
\end{aligned}
$$

Proof. It is obvious from definition that for any $u, v \in V\left(G_{1}+G_{2}\right)$, the distance $d_{G_{1}+G_{2}}(u, v)$ is either 1 or 2. In the formula for $H_{\lambda}\left(G_{1}+G_{2}\right)$, we partition the set of pairs of vertices of $G_{1}+G_{2}$ into three subsets $A_{0}, A_{1}$ and $A_{2}$. In $A_{0}$ we collect all pairs of vertices $u$ and $v$ such that $u$ is in $G_{1}$ and $v$ is in $G_{2}$. Hence, they are adjacent in $G_{1}+G_{2}$. The set $A_{i}, i=1,2$ is the set of pairs of vertices $u$ and $v$ which are in $G_{i}$. Also we partition the sum in the formula of $H_{\lambda}\left(G_{1}+G_{2}\right)$ into three sums $S_{i}$ so that $S_{i}$ is over $A_{i}$ for $i=0,1,2$. For $S_{0}$ we obtain

$$
\begin{aligned}
S_{0} & =\sum_{u \in V\left(G_{1}\right)} \sum_{v \in V\left(G_{2}\right)}\left(d_{G_{1}}(u)+d_{G_{2}}(v)+n_{1}+n_{2}\right) \\
& =n_{1} n_{2}\left(n_{1}+n_{2}\right)+2 n_{2} m_{1}+2 n_{1} m_{2},
\end{aligned}
$$

and

$$
\begin{aligned}
S_{1}= & \sum_{\{u, v\} \subseteq V\left(G_{1}\right)}\left(d_{G_{1}}(u)+d_{G_{1}}(v)+2 n_{2}\right) d_{G_{1}+G_{2}}^{\lambda}(u, v) \\
= & \sum_{u v \in E\left(G_{1}\right)}\left(d_{G_{1}}(u)+d_{G_{2}}(v)+2 n_{2}\right) \\
& +\sum_{u v \notin E\left(G_{1}\right)} 2^{\lambda}\left(d_{G_{1}}(u)+d_{G_{2}}(v)+2 n_{2}\right) \\
& =M_{1}\left(G_{1}\right)+2 n_{2} m_{1}+2^{\lambda} \bar{M}_{1}\left(G_{1}\right)+2^{\lambda+1} n_{2} \bar{m}_{1} .
\end{aligned}
$$

Similarly,

$$
S_{2}=M_{1}\left(G_{2}\right)+2 n_{1} m_{2}+2^{\lambda} \bar{M}_{1}\left(G_{2}\right)+2^{\lambda+1} n_{1} \bar{m}_{2} .
$$

Therefore

$$
\begin{aligned}
& H_{\lambda}\left(G_{1}+G_{2}\right) \\
= & S_{0}+S_{1}+S_{2} \\
= & n_{1} n_{2}\left(n_{1}+n_{2}\right)+4 n_{2} m_{1}+4 n_{1} m_{2}+M_{1}\left(G_{1}\right)+M_{1}\left(G_{2}\right) \\
& +2^{\lambda}\left(\bar{M}_{1}\left(G_{1}\right)+\bar{M}_{1}\left(G_{2}\right)\right)+2^{\lambda+1}\left(n_{2} \bar{m}_{1}+n_{1} \bar{m}_{2}\right) .
\end{aligned}
$$

Corollary 2.3. Let $G$ be a connected graph with $n$ vertices and $m$ edges. Then

$$
\begin{aligned}
H_{\lambda}\left(K_{1}+G\right)= & n(n+1)+4 m+M_{1}(G) \\
& +2^{\lambda} \bar{M}_{1}(G)+2^{\lambda+1} \bar{m}
\end{aligned} .
$$

The exact formulas $H_{\lambda}(G)$ for the fan graph $K_{1}+P_{n}$ and for the wheel graph $W_{n}=K_{1}+C_{n}$ are given in the following Corollary.

\section{Corollary 2.4.}

$$
H_{\lambda}\left(K_{1}+P_{n}\right)=n_{2}+9 n-10+2^{\lambda}(n-2)(5 n-9),
$$

and

$$
H_{\lambda}\left(K_{1}+C_{n}\right)=n_{2}+9 n+3 \times 2^{\lambda} n(n-3) .
$$

Remark 2.5. In the above theorem, if $\lambda=1$, then we obtain $D^{\prime}\left(G_{1}+G_{2}\right)$, which gives first derivatives formula Theorem 3 in [22] at $x=1$.

In the next theorem we obtain the exact formula for the generalized degree distance of the composition of two graphs.

Theorem 2.6. Let $G_{1}$ and $G_{2}$ be two graphs. Then 


$$
\begin{aligned}
& H_{\lambda}\left(G_{1}\left[G_{2}\right]\right)=4 m_{2} m_{1} n_{2}+n_{1} M_{1}\left(G_{2}\right)+2^{\lambda+2} \bar{m}_{2} n_{2} m_{1} \\
& +2^{\lambda} n_{1} \bar{M}_{1}\left(G_{2}\right)+n_{2}^{3} H_{\lambda}\left(G_{1}\right)+4 n_{2} m_{2} W_{\lambda}\left(G_{1}\right) \\
& H_{\lambda}\left(G_{1}\left[G_{2}\right]\right)=\sum_{\{u, v\} \subseteq V\left(G_{1}\left[G_{2}\right]\right)} d_{G_{1}\left[G_{2}\right]}^{\lambda}(u, v)\left(d_{G_{1}\left[G_{2}\right]}(u)+d_{G_{1}\left[G_{2}\right]}(v)\right) \\
& =\frac{1}{2} \sum_{\left(u_{i}, v_{k}\right)} \sum_{\left(u_{j}, v_{l}\right)} d_{G_{1}\left[G_{2}\right]}^{\lambda}\left(\left(u_{i}, v_{k}\right),\left(u_{j}, v_{l}\right)\right)\left(n d_{G_{1}}\left(u_{i}\right)+d_{G_{2}}\left(v_{k}\right)+n d_{G_{1}}\left(u_{j}\right)+d_{G_{2}}\left(v_{l}\right)\right) \\
& =\sum_{p=1}^{n_{1}} \sum_{k, l=1}^{n_{2}} d_{G_{1}\left[G_{2}\right]}^{\lambda}\left(\left(u_{p}, v_{k}\right),\left(u_{p}, v_{l}\right)\right)\left(2 n_{2} d_{G_{1}}\left(u_{p}\right)+d_{G_{2}}\left(v_{k}\right)+d_{G_{2}}\left(v_{l}\right)\right) \\
& \left.+\sum_{k, l=1, j=1, i \neq j}^{n_{2}} \sum_{G_{1}\left[G_{2}\right]}^{n_{1}} d_{i}^{\lambda}\left(u_{i}, v_{k}\right),\left(u_{j}, v_{l}\right)\right)\left(n_{2}\left(d_{G_{1}}\left(u_{i}\right)+d_{G_{1}}\left(u_{j}\right)\right)+d_{G_{2}}\left(v_{k}\right)+d_{G_{2}}\left(v_{l}\right)\right) \\
& +\sum_{p=1}^{n_{1}} \sum_{k, l=1, u_{k} v_{l} \notin E\left(G_{2}\right)}^{n_{2}} 2^{\lambda}\left(2 n_{2} d_{G_{1}}\left(u_{p}\right)+d_{G_{2}}\left(v_{k}\right)+d_{G_{2}}\left(v_{l}\right)\right)+n_{2}^{3} H_{\lambda}\left(G_{1}\right)+4 n_{2} m_{2} W_{\lambda}\left(G_{1}\right) \\
& =4 m_{2} m_{1} n_{2}+n_{1} M_{1}\left(G_{2}\right)+2^{\lambda+2} \bar{m}_{2} n_{2} m_{1}+2^{\lambda} n_{1} \bar{M}_{1}\left(G_{2}\right)+n_{2}^{3} H_{\lambda}\left(G_{1}\right)+4 n_{2} m_{2} W_{\lambda}\left(G_{1}\right) \text {. }
\end{aligned}
$$

Proof. Suppose $\left\{u_{1}, \cdots, u_{n_{1}}\right\}$ and $\left\{v_{1}, \cdots, v_{n_{2}}\right\}$ are two set of vertices of $G_{1}$ and $G_{2}$, respectively. Then

So the proof of theorem is now completed. $\square$

By composing paths and cycles with various small graphs we can obtain classes of polymer-like graphs. Now we give the formula of the $H_{\lambda}$ index for the fence graph $P_{n}\left[K_{2}\right]$ and the closed fence $C_{n}\left(K_{2}\right)$.

\section{Corollary 2.7.}

$$
H_{\lambda}\left(P_{n}\left[K_{2}\right]\right)=9 n-8+8\left(H_{\lambda}\left(P_{n}\right)+W_{\lambda}\left(P_{n}\right)\right),
$$

and

$$
H_{\lambda}\left(C_{n}\left[K_{2}\right]\right)=9 n+8\left(H_{\lambda}\left(C_{n}\right)+W_{\lambda}\left(C_{n}\right)\right) .
$$

Remark 2.8. In Theorem 2.6, if $\lambda=1$, then we obtain $D^{\prime}\left(G_{1}\left[G_{2}\right]\right)$, which gives first derivatives formula Theorem 5 in [22] at $x=1$.

Now we prove the theorem that characterizes the generalized degree distance of the disjunction of two graphs.

Theorem 2.9. Let $G_{1}$ and $G_{2}$ be two graphs. Then

$$
\begin{aligned}
H_{\lambda}\left(G_{1} \vee G_{2}\right)= & 8 n_{1} n_{2} m_{1} m_{2}+\left(n_{1}^{3}-4 n_{1} m_{1}\right) M_{1}\left(G_{2}\right)+\left(n_{2}{ }^{3}-4 n_{2} m_{2}\right) M_{1}\left(G_{1}\right)+M_{1}\left(G_{1}\right) M_{1}\left(G_{2}\right) \\
& +2^{\lambda}\left[\left(2 n_{2} \bar{m}_{2}+n_{2}{ }^{2}-2 m_{2}\right) \bar{M}_{1}\left(G_{1}\right)+\left(2 n_{1} \bar{m}_{1}+n_{1}{ }^{2}-2 m_{1}\right) \bar{M}_{1}\left(G_{2}\right)\right] \\
& -2^{\lambda} \bar{M}_{1}\left(G_{1}\right) \bar{M}_{1}\left(G_{2}\right)+2^{\lambda+2}\left(n_{1} \bar{m}_{1} m_{2}+n_{2} \bar{m}_{2} m_{1}\right)
\end{aligned}
$$

Proof. According to definition of $G_{1} \vee G_{2}$, we have the following relations:

$$
\begin{aligned}
S_{1} & =\sum_{\{u, v\} \subseteq V\left(G_{1}\right)} \sum_{u v \in E\left(G_{2}\right)}\left(n_{2} d_{G_{1}}(x)+n_{1} d_{G_{2}}(u)-d_{G_{1}}(x) d_{G_{2}}(u)+n_{2} d_{G_{1}}(y)+n_{1} d_{G_{2}}(v)-d_{G_{1}}(y) d_{G_{2}}(v)\right) \\
& =\sum_{\{x, y\} \subseteq V\left(G_{1}\right)} \sum_{u v \in E\left(G_{2}\right)}\left(n_{1}\left(d_{G_{2}}(u)+d_{G_{2}}(v)\right)+n_{2}\left(d_{G_{1}}(y)+d_{G_{1}}(x)\right)-d_{G_{1}}(x) d_{G_{2}}(u)-d_{G_{1}}(y) d_{G_{2}}(v)\right) \\
& =n_{1}^{3} M_{1}\left(G_{2}\right)+4 n_{1} m_{2} m_{1} n_{2}-2 n_{1} m_{1} M_{1}\left(G_{2}\right), \\
S_{2} & =\sum_{x y \in E\left(G_{1}\right)\{u, v\} \in V\left(G_{2}\right)}\left(n_{2} d_{G_{1}}(x)+n_{1} d_{G_{2}}(u)-d_{G_{1}}(x) d_{G_{2}}(u)+n_{2} d_{G_{1}}(y)+n_{1} d_{G_{2}}(v)-d_{G_{1}}(y) d_{G_{2}}(v)\right) \\
& =n_{2}^{3} M_{1}\left(G_{1}\right)+4 n_{1} m_{2} m_{1} n_{2}-2 n_{2} m_{2} M_{1}\left(G_{1}\right), \\
& S_{3}=\sum_{x y \in E\left(G_{1}\right)} \sum_{u v \in E\left(G_{1}\right)}\left(n_{2} d_{G_{1}}(x)+n_{1} d_{G_{2}}(u)-d_{G_{1}}(x) d_{G_{2}}(u)+n_{2} d_{G_{1}}(y)+n_{1} d_{G_{2}}(v)-d_{G_{1}}(y) d_{G_{2}}(v)\right) \\
& =2 n_{1} m_{1} M_{1}\left(G_{2}\right)+2 n_{2} m_{2} M_{1}\left(G_{1}\right)-M_{1}\left(G_{1}\right) M_{1}\left(G_{2}\right),
\end{aligned}
$$

and 


$$
\begin{aligned}
S_{4} & =\sum_{x y \notin E\left(G_{1}\right), x \neq y} \sum_{u v \notin E\left(G_{2}\right), u \neq v} 2^{\lambda}\left(n_{2} d_{G_{1}}(x)+n_{1} d_{G_{2}}(u)-d_{G_{1}}(x) d_{G_{2}}(u)+n_{2} d_{G_{1}}(y)+n_{1} d_{G_{2}}(v)-d_{G_{1}}(y) d_{G_{2}}(v)\right) \\
& +2^{\lambda} \sum_{x y \notin E\left(G_{1}\right)} \sum_{u \in V\left(G_{2}\right)}\left(2 n_{1} d_{G_{2}}(u)+\left(n_{2}-d_{G_{2}}(u)\right)\left(d_{G_{1}}(x)+d_{G_{1}}(y)\right)\right) \\
& +2^{\lambda} \sum_{x \in V\left(G_{1}\right)} \sum_{u v \notin E\left(G_{2}\right)}\left(2 n_{2} d_{G_{1}}(x)+\left(n_{1}-d_{G_{1}}(x)\right)\left(d_{G_{2}}(u)+d_{G_{2}}(v)\right)\right) \\
& =2^{\lambda}\left[\left(2 n_{2} \bar{m}_{2}+n_{2}^{2}-2 m_{2}\right) \bar{M}_{1}\left(G_{1}\right)+\left(2 n_{1} \bar{m}_{1}+n_{1}^{2}-2 m_{1}\right) \bar{M}_{1}\left(G_{2}\right)-\bar{M}_{1}\left(G_{1}\right) \bar{M}_{1}\left(G_{2}\right)\right]+2^{\lambda+2}\left(n_{1} \bar{m}_{1} m_{2}+n_{2} \bar{m}_{2} m_{1}\right) .
\end{aligned}
$$

So we have:

$$
\begin{aligned}
& H_{\lambda}\left(G_{1} \vee G_{2}\right) \\
& =S_{1}+S_{2}+S_{4}-S_{3}=8 n_{1} n_{2} m_{1} m_{2}+\left(n_{1}^{3}-4 n_{1} m_{1}\right) M_{1}\left(G_{2}\right)+\left(n_{2}^{3}-4 n_{2} m_{2}\right) M_{1}\left(G_{1}\right)+M_{1}\left(G_{1}\right) M_{1}\left(G_{2}\right) \\
& +2^{\lambda}\left[\left(2 n_{2} \bar{m}_{2}+n_{2}^{2}-2 m_{2}\right) \bar{M}_{1}\left(G_{1}\right)+\left(2 n_{1} \bar{m}_{1}+n_{1}^{2}-2 m_{1}\right) \bar{M}_{1}\left(G_{2}\right)\right]-2^{\lambda} \bar{M}_{1}\left(G_{1}\right) \bar{M}_{1}\left(G_{2}\right)+2^{\lambda+2}\left(n_{1} \bar{m}_{1} m_{2}+n_{2} \bar{m}_{2} m_{1}\right) .
\end{aligned}
$$

This completes the proof. $\square$

Now we prove the theorem that characterizes the generalized degree distance of the symmetric difference of two graphs.

Theorem 2.10. Let $G_{1}$ and $G_{2}$ be two graphs. Then

$$
\begin{aligned}
H_{\lambda}\left(G_{1} \oplus G_{2}\right)= & 8 n_{1} n_{2} m_{1} m_{2}+\left(n_{1}^{3}-8 n_{1} m_{1}\right) M_{1}\left(G_{2}\right)+\left(n_{2}{ }^{3}-8 n_{2} m_{2}\right) M_{1}\left(G_{1}\right)+4 M_{1}\left(G_{1}\right) M_{1}\left(G_{2}\right) \\
& +2^{\lambda}\left[\left(2 n_{2} \bar{m}_{2}+n_{2}{ }^{2}-4 m_{2}\right) \bar{M}_{1}\left(G_{1}\right)+\left(2 n_{1} \bar{m}_{1}+n_{1}{ }^{2}-4 m_{1}\right) \bar{M}_{1}\left(G_{2}\right)\right] \\
& -2^{\lambda} \bar{M}_{1}\left(G_{1}\right) \bar{M}_{1}\left(G_{2}\right)+2^{\lambda+2}\left(n_{1} \bar{m}_{1} m_{2}+n_{2} \bar{m}_{2} m_{1}\right)
\end{aligned}
$$

Proof. We consider four sums $S_{1}, \cdots, S_{4}$ as follows:

$$
\begin{aligned}
S_{1} & =\sum_{\{x, y\} \subseteq V\left(G_{1}\right)} \sum_{u v \in E\left(G_{2}\right)}\left(n_{2} d_{G_{1}}(x)+n_{1} d_{G_{2}}(u)-2 d_{G_{1}}(x) d_{G_{2}}(u)+n_{2} d_{G_{1}}(y)+n_{1} d_{G_{2}}(v)-2 d_{G_{1}}(y) d_{G_{2}}(v)\right) \\
& =n_{1}^{3} M_{1}\left(G_{2}\right)+4 n_{1} m_{2} m_{1} n_{2}-4 n_{1} m_{1} M_{1}\left(G_{2}\right),
\end{aligned}
$$

similarly to $S_{1}$

$$
\begin{aligned}
S_{2} & =n_{2}^{3} M_{1}\left(G_{1}\right)+4 n_{1} m_{2} m_{1} n_{2}-4 n_{2} m_{2} M_{1}\left(G_{1}\right), \\
S_{3} & =\sum_{x y \in E\left(G_{1}\right)} \sum_{u v \in E\left(G_{2}\right)}\left(n_{2} d_{G_{1}}(x)+n_{1} d_{G_{2}}(u)-2 d_{G_{1}}(x) d_{G_{2}}(u)+n_{2} d_{G_{1}}(y)+n_{1} d_{G_{2}}(v)-2 d_{G_{1}}(y) d_{G_{2}}(v)\right) \\
& =2 n_{1} m_{1} M_{1}\left(G_{2}\right)+2 n_{2} m_{2} M_{1}\left(G_{1}\right)-2 M_{1}\left(G_{1}\right) M_{1}\left(G_{2}\right),
\end{aligned}
$$

and

$$
\begin{aligned}
S_{4} & =\sum_{x y \notin E\left(G_{1}\right), x \neq y} \sum_{u v \notin E\left(G_{2}\right), u \neq v} 2^{\lambda}\left(n_{2} d_{G_{1}}(x)+n_{1} d_{G_{2}}(u)-2 d_{G_{1}}(x) d_{G_{2}}(u)+n_{2} d_{G_{1}}(y)+n_{1} d_{G_{2}}(v)-2 d_{G_{1}}(y) d_{G_{2}}(v)\right) \\
& +2^{\lambda} \sum_{x y \notin E\left(G_{1}\right)} \sum_{u \in V\left(G_{2}\right)}\left(2 n_{1} d_{G_{2}}(u)+\left(n_{2}-2 d_{G_{2}}(u)\right)\left(d_{G_{1}}(x)+d_{G_{1}}(y)\right)\right) \\
& +2^{\lambda} \sum_{x \in V\left(G_{1}\right)} \sum_{u v \notin E\left(G_{2}\right)}\left(2 n_{2} d_{G_{1}}(x)+\left(n_{1}-2 d_{G_{1}}(x)\right)\left(d_{G_{2}}(u)+d_{G_{2}}(v)\right)\right) \\
& =2^{\lambda}\left[\left(2 n_{2} \bar{m}_{2}+n_{2}^{2}-4 m_{2}\right) \bar{M}_{1}\left(G_{1}\right)+\left(2 n_{1} \bar{m}_{1}+n_{1}^{2}-4 m_{1}\right) \bar{M}_{1}\left(G_{2}\right)-2 \bar{M}_{1}\left(G_{1}\right) \bar{M}_{1}\left(G_{2}\right)\right] \\
& +2^{\lambda+2}\left(n_{1} \bar{m}_{1} m_{2}+n_{2} \bar{m}_{2} m_{1}\right) .
\end{aligned}
$$

By the definition of $G_{1} \oplus G_{2}$, we have:

$$
\begin{aligned}
H_{\lambda}\left(G_{1} \oplus G_{2}\right)= & S_{1}+S_{2}+S_{4}-2 S_{3}=8 n_{1} n_{2} m_{1} m_{2}+\left(n_{1}^{3}-8 n_{1} m_{1}\right) M_{1}\left(G_{2}\right)+\left(n_{2}^{3}-8 n_{2} m_{2}\right) M_{1}\left(G_{1}\right)+4 M_{1}\left(G_{1}\right) M_{1}\left(G_{2}\right) \\
& +2^{\lambda}\left[\left(2 n_{2} \bar{m}_{2}+n_{2}^{2}-4 m_{2}\right) \bar{M}_{1}\left(G_{1}\right)+\left(2 n_{1} \bar{m}_{1}+n_{1}^{2}-4 m_{1}\right) \bar{M}_{1}\left(G_{2}\right)-2 \bar{M}_{1}\left(G_{1}\right) \bar{M}_{1}\left(G_{2}\right)\right] \\
& +2^{\lambda+2}\left(n_{1} \bar{m}_{1} m_{2}+n_{2} \bar{m}_{2} m_{1}\right) .
\end{aligned}
$$

So the proof is now completed. $\square$ 
In the next theorem we find the generalized degree distance of the Cartesian product of two graphs.

Theorem 2.11. Let $G_{1}$ and $G_{2}$ be two graphs. Then

$$
\begin{aligned}
H_{\lambda}\left(G_{1} \times G_{2}\right)= & n_{1}^{2} H_{\lambda}\left(G_{2}\right) 4 m_{1} n_{1} W_{\lambda}\left(G_{2}\right)+2\left(\begin{array}{c}
\lambda \\
1
\end{array}\right)\left(H_{1}\left(G_{1}\right) W_{\lambda-1}\left(G_{2}\right)+W\left(G_{1}\right) H_{\lambda-1}\left(G_{2}\right)\right) \\
& +2\left(\begin{array}{c}
\lambda \\
2
\end{array}\right)\left(H_{2}\left(G_{1}\right) W_{\lambda-2}\left(G_{2}\right)+W_{2}\left(G_{1}\right) H_{\lambda-2}\left(G_{2}\right)\right)+\cdots+2\left(\begin{array}{c}
\lambda \\
\lambda-1
\end{array}\right)\left(H_{\lambda-1}\left(G_{1}\right) W\left(G_{2}\right)+W_{\lambda-1}\left(G_{1}\right) H_{1}\left(G_{2}\right)\right) \\
& +n_{2}^{2} H_{\lambda}\left(G_{1}\right)+4 m_{2} n_{2} W_{\lambda}\left(G_{1}\right) .
\end{aligned}
$$

Proof. Suppose $\left\{u_{1}, \cdots, u_{n_{1}}\right\}$ and $\left\{v_{1}, \cdots, v_{n_{2}}\right\}$ are two set of vertices of $G_{1}$ and $G_{2}$, respectively. Then by Lemma 2.1 and definition of $H_{\lambda}$, we have:

$$
\begin{aligned}
H_{\lambda}\left(G_{1} \times G_{2}\right)= & \sum_{\{u, v\} \subseteq V\left(G_{1} \times G_{2}\right)} d_{G_{1} \times G_{2}}^{\lambda}(u, v)\left(d_{G_{1} \times G_{2}}(u)+d_{G_{1} \times G_{2}}(v)\right) \\
= & \frac{1}{2} \sum_{\left(u_{i}, v_{k}\right)} \sum_{\left(u_{j}, v_{l}\right)} d_{G_{1} \times G_{2}}^{\lambda}\left(\left(u_{i}, v_{k}\right),\left(u_{j}, v_{l}\right)\right)\left(d_{G_{1}}\left(u_{i}\right)+d_{G_{2}}\left(v_{k}\right)+d_{G_{1}}\left(u_{j}\right)+d_{G_{2}}\left(v_{l}\right)\right) \\
= & \frac{1}{2} \sum_{k, l=1}^{n_{2}} \sum_{i, j=1}^{n_{1}}\left(d_{G_{1}}\left(u_{i}, u_{j}\right)+d_{G_{2}}\left(v_{k}, v_{l}\right)\right)^{\lambda}\left(d_{G_{1}}\left(u_{i}\right)+d_{G_{2}}\left(v_{k}\right)+d_{G_{1}}\left(u_{j}\right)+d_{G_{2}}\left(v_{l}\right)\right) \\
= & \frac{1}{2} \sum_{k, l=1}^{n_{2}} \sum_{i, j=1}^{n_{1}}\left(\sum_{r=0}^{\lambda} \lambda_{r} d_{G_{1}}^{r}\left(u_{i}, u_{j}\right) d_{G_{2}}^{\lambda-r}\left(v_{k}, v_{l}\right)\right)\left(d_{G_{1}}\left(u_{i}\right)+d_{G_{2}}\left(v_{k}\right)+d_{G_{1}}\left(u_{j}\right)+d_{G_{2}}\left(v_{l}\right)\right) \\
= & n_{1}^{2} H_{\lambda}\left(G_{2}\right)+4 m_{1} n_{1} W_{\lambda}\left(G_{2}\right)+2\left(\begin{array}{c}
\lambda \\
1
\end{array}\right)\left(H_{1}\left(G_{1}\right) W_{\lambda-1}\left(G_{2}\right)+W\left(G_{1}\right) H_{\lambda-1}\left(G_{2}\right)\right) \\
& +2\left(\begin{array}{c}
\lambda \\
2
\end{array}\right)\left(H_{2}\left(G_{1}\right) W_{\lambda-2}\left(G_{2}\right)+W_{2}\left(G_{1}\right) H_{\lambda-2}\left(G_{2}\right)\right) \\
& +\cdots+2\left(\begin{array}{c}
\lambda \\
\lambda-1
\end{array}\right)\left(H_{\lambda-1}\left(G_{1}\right) W\left(G_{2}\right)+W_{\lambda-1}\left(G_{1}\right) H_{1}\left(G_{2}\right)\right)+n_{2}^{2} H_{\lambda}\left(G_{1}\right)+4 m_{2} n_{2} W_{\lambda}\left(G_{1}\right) .
\end{aligned}
$$

So the proof is now completed. $\square$

As an application of the above theorem, we list explicit formulae for the generalized degree distance of $P_{n} \times P_{m}, P_{n} \times C_{m}$ and $C_{n} \times C_{m}$. These graphs are known as the rectangular grid, the $C_{4}$ nanotube, and the $C_{4}$ nanotorus, respectively.

Lemma 2.12. Define $\alpha(k, r)=\sum_{i=1}^{k} i^{r}$. By [1,23], we have:

$$
\begin{aligned}
& W_{\lambda}\left(P_{n}\right)=n \alpha(n-1, \lambda)-\alpha(n-1, \lambda+1), \\
& W_{\lambda}\left(C_{n}\right)=\left\{\begin{array}{l}
n \alpha\left(\frac{n}{2}-1, \lambda\right)+\left(\frac{n}{2}\right)^{\lambda} \frac{n}{2}, n \text { is even } \\
n \alpha\left(\frac{n-1}{2}, \lambda\right),
\end{array},\right. \\
& H_{\lambda}\left(P_{n}\right)=-2 \alpha(n-3, \lambda)+4 n \alpha(n-3, \lambda) \\
& -4 \alpha(n-3, \lambda+1)+2(n-1)^{\lambda}+6(n-2)^{\lambda}, \\
& H_{\lambda}\left(C_{n}\right)=\left\{\begin{array}{l}
4 n \alpha\left(\frac{n}{2}-1, \lambda\right)+\left(\frac{n}{2}\right)^{\lambda+1}, n \text { is even } \\
4 n \alpha\left(\frac{n-1}{2}, \lambda\right),
\end{array},\right.
\end{aligned}
$$

Corollary 2.13. By Theorem 2.9 and Lemma 2.12 we have:

$$
\begin{aligned}
& H_{\lambda}\left(C_{n} \times C_{m}\right) \\
= & n^{2} H_{\lambda}\left(C_{m}\right)+4 n^{2} W_{\lambda}\left(C_{m}\right)+m^{2} H_{\lambda}\left(C_{n}\right)+4 m^{2} W_{\lambda}\left(C_{n}\right) \\
+ & 2 \sum_{i=1}^{\lambda-1}\left(\begin{array}{c}
\lambda \\
i
\end{array}\right)\left(H_{i}\left(C_{n}\right) W_{\lambda-i}\left(C_{m}\right)+H_{\lambda-i}\left(C_{n}\right) W_{i}\left(C_{m}\right)\right), \\
& H_{\lambda}\left(P_{n} \times P_{m}\right) \\
= & n^{2} H_{\lambda}\left(P_{m}\right)+4 n(n-1) W_{\lambda}\left(P_{m}\right)+m^{2} H_{\lambda}\left(P_{n}\right) \\
+ & 4 m(m-1) W_{\lambda}\left(P_{n}\right) \\
+ & 2 \sum_{i=1}^{\lambda-1}\left(\begin{array}{c}
\lambda \\
i
\end{array}\right)\left(H_{i}\left(P_{n}\right) W_{\lambda-i}\left(P_{m}\right)+H_{\lambda-i}\left(P_{n}\right) W_{i}\left(P_{m}\right)\right), \\
\text { and } \quad & H_{\lambda}\left(P_{n} \times C_{m}\right) \\
= & n^{2} H_{\lambda}\left(C_{m}\right)+4 n(n-1) W_{\lambda}\left(C_{m}\right) \\
& +m^{2} H_{\lambda}\left(P_{n}\right)+4 m^{2} W_{\lambda}\left(P_{n}\right) \\
& +2 \sum_{i=1}^{\lambda-1}\left(\begin{array}{c}
\lambda \\
i
\end{array}\right)\left(H_{i}\left(P_{n}\right) W_{\lambda-i}\left(C_{m}\right)+H_{\lambda-i}\left(P_{n}\right) W_{i}\left(C_{m}\right)\right) .
\end{aligned}
$$

and

Remark 2.14. In the above theorem, if $\lambda=1$, then we obtain $D^{\prime}\left(G_{1} \times G_{2}\right)$, which gives first derivatives for- 
mula Theorem 1 in [22] at $x=1$.

Now we obtain the relation between the generalized degree distance polynomial and Wiener-type invariant polynomial and the relation between the modified generalized degree distance polynomial and Wiener-type invariant polynomial for graphs.

Theorem 2.15. If $G$ is a graph with $n$ vertices and $m$ edges, then

$$
\begin{aligned}
H_{\lambda}(G, x)= & \sum_{u \neq v \in V(G)}\left(d_{G}(u)+d_{G}(v)-2\right) x^{d^{\lambda}(u, v)} \\
& +4 m-2 n+2 W_{\lambda}(G, x) .
\end{aligned}
$$

Proof. By definition, we have

$$
\begin{aligned}
H_{\lambda}(G, x)= & \sum_{\{u, v\} \subseteq V(G)}\left(d_{G}(u)+d_{G}(v)\right) x^{d^{\lambda}(u, v)} \\
= & \sum_{u \neq v \in V(G)}\left(d_{G}(u)+d_{G}(v)-2\right) x^{d^{\lambda}(u, v)} \\
& +\sum_{u=v \in V(G)}\left(d_{G}(u)+d_{G}(v)-2\right) x^{d^{\lambda}(u, v)} \\
& +2 \sum_{\{u, v\} \subseteq V(G)} x^{d^{\lambda}(u, v)} \\
& =\sum_{u \neq v \in V(G)}\left(d_{G}(u)+d_{G}(v)-2\right) x^{d^{\lambda}(u, v)} \\
& +4 m-2 n+2 W_{\lambda}(G, x) .
\end{aligned}
$$

This completes the proof. $\square$

Theorem 2.16. If $G$ is a graph with $n$ vertices and $m$ edges, then

$$
\begin{aligned}
H_{\lambda}^{*}(G, x)= & \sum_{u \neq v \in V(G)}\left(d_{G}(u)-1\right)\left(d_{G}(v)-1\right) x^{d^{\lambda}(u, v)} \\
& +M_{1}(G)-4 m+n+H_{\lambda}(G, x)-W_{\lambda}(G, x) .
\end{aligned}
$$

Proof. By definition, we have

$$
\begin{aligned}
& H_{\lambda}^{*}(G, x) \\
& =\sum_{\{u, v\} \subseteq V(G)}\left(d_{G}(u) d_{G}(v)\right) x^{d^{\lambda}(u, v)} \\
& =\sum_{u \neq v \in V(G)}\left(d_{G}(u)-1\right)\left(d_{G}(v)-1\right) x^{d^{\lambda}(u, v)} \\
& +\sum_{u=v \in V(G)}\left(d_{G}(u)-1\right)\left(d_{G}(v)-1\right) x^{d^{\lambda}(u, v)} \\
& +\sum_{\{u, v\} \subseteq V(G)}\left(d_{G}(u)+d_{G}(v)\right) x^{d^{\lambda}(u, v)}-\sum_{\{u, v\} \subseteq V(G)} x^{d^{\lambda}(u, v)} \\
& =\sum_{u \neq v \in V(G)}\left(d_{G}(u)-1\right)\left(d_{G}(v)-1\right) x^{d^{\lambda}(u, v)} \\
& +\sum_{u \in V(G)} d_{G}^{2}(u)-4 m+n+H_{\lambda}(G, x)-W_{\lambda}(G, x) \\
& =\sum_{u \neq v \in V(G)}\left(d_{G}(u)-1\right)\left(d_{G}(v)-1\right) x^{d^{\lambda}(u, v)} \\
& +M_{1}(G)-4 m+n+H_{\lambda}(G, x)-W_{\lambda}(G, x) .
\end{aligned}
$$

This completes the proof. $\square$

\section{REFERENCES}

[1] A. Hamzeh, A. Iranmanesh, S. Hossein-Zadeh and M. V. Diudea, "Generalized Degree Distance of Trees, Unicyclic and Bicyclic Graphs," Studia Ubb Chemia, LVII, Vol. 4, 2012, pp. 73-85.

[2] M. V. Diudea, I. Gutman and L. Jantschi, "Molecular Topology," Nova Science, Huntington, 2001.

[3] W. Imrich and S. Klavžar, "Product Graphs: Structure and Recognition," John Wiley \& Sons, New York, 2000.

[4] I. Gutman and N. Trinajstic, "Graph Theory and Molecular Orbitals. Total $\pi$-Electron Energy of Alternant Hydrocarbons," Chemical Physics Letters, Vol. 17, No. 4, 1972, pp. 535-538. doi:10.1016/0009-2614(72)85099-1

[5] S. Nikolic, G. Kovacevic, A. Milicevic and N. Trinajstic, "The Zagreb Indices 30 Years after," Croatica Chemica Acta, Vol. 76, No. 2, 2003, pp. 113-124.

[6] T. Došlic, "Vertex-Weighted Wiener Polynomials for Composite Graphs," Ars Mathematica Contemporanea, Vol. 1, No. 1, 2008, pp. 66-80.

[7] I. Gutman, "A Property of the Wiener Number and Its Modifications," Indian Journal of Chemistry, Vol. 36A, No. 2, 1997, pp. 128-132.

[8] I. Gutman, A. A. Dobrynin, S. Klavžar and L. Pavlovic, "Wiener-Type Invariants of Trees and Their Relation," Bulletin of the Institute of Combinatorics and Its Applications, Vol. 40, No. 1, 2004, pp. 23-30.

[9] Y. Alizadeh, A. Iranmanesh and T. Došlic, "Additively Weighted Harary Index of Some Composite Graphs," Discrete Mathematics, Vol. 313, No. 1, 2013, pp. 26-34. doi:10.1016/j.disc.2012.09.011

[10] A. A. Dobrynin and A. A. Kochetova, "Degree Distance of a Graph: A Degree Analogue of the Wiener Index," Journal of Chemical Information and Computer Sciences, Vol. 34, No. 5, 1994, pp. 1082-1086. doi: $10.1021 / \mathrm{ci00021a008}$

[11] I. Gutman, "Selected Properties of the Schultz Molecular Topological Index," Journal of Chemical Information and Computer Sciences, Vol. 34, No. 5, 1994, pp. 1087-1089. doi:10.1021/ci00021a009

[12] I. Gutman, and S. Klavžar, "Wiener Number of VertexWeighted Graphs and a Chemical Applications," Discrete Applied Mathematics, Vol. 80, No. 1, 1997, pp. 73-81. doi:10.1016/S0166-218X(97)00070-X

[13] H. Hua and S. Zhang, "On the Reciprocal Degree Distance of Graphs," Discrete Applied Mathematics, Vol. 160, No. 7-8, 2012, pp. 1152-1163. doi:10.1016/j.dam.2011.11.032

[14] S. Hossein-Zadeh, A. Hamzeh and A. R. Ashrafi, "Extremal Properties of Zagreb Coindices and Degree Distance of Graphs," Miskolc Mathematical Notes, Vol. 11, No. 2, 2010, pp. 129-137.

[15] I. Tomescu, "Unicyclic and Bicyclic Graphs Having Minimum Degree Distance," Discrete Applied Mathematics, Vol. 156, No. 1, 2008, pp. 125-130. doi:10.1016/j.dam.2007.09.010 
[16] I. Tomescu, "Some Extremal Properties of the Degree Distance of a Graph," Discrete Applied Mathematics, Vol. 98, No. 1-2, 1999, pp. 159-163. doi:10.1016/S0166-218X(99)00117-1

[17] A. Graovac and T. Pisanski, "On the Wiener Index of a Graph," Journal of Mathematical Chemistry, Vol. 8, No. 1, 1991, pp. 53-62. doi:10.1007/BF01166923

[18] B. E. Sagan, Y.-N. Yeh and P. Zhang, "The Wiener Polynomial of a Graph," International Journal of Quantum Chemistry, Vol. 60, No. 5, 1996, pp. 959-969. doi:10.1002/(SICI)1097-461X(1996)60:5<959::AID-QU A2>3.0.CO;2-W

[19] S. Klavžar, A. Rajapakse and I. Gutman, "The Szeged and the Wiener Index of Graphs," Applied Mathematics Letters, Vol. 9, No. 5, 1996, pp. 45-49. doi:10.1016/0893-9659(96)00071-7

[20] M. H. Khalifeh, H. Yousefi-Azari and A. R. Ashrafi, "The Hyper-Wiener Index of Graph Operations," Computers \& Mathematics with Applications, Vol. 56, No. 5, 2008, pp. 1402-1407. doi:10.1016/j.camwa.2008.03.003
[21] M. Eliasi and A. Iranmanesh, "The Hyper-Wiener Index of the Generalized Hierarchical Product of Graphs," Discrete Applied Mathematics, Vol. 159, No. 8, 2011, pp. 866-871. doi:10.1016/j.dam.2010.12.020

[22] M. Eliasi and B. Taeri, "Schultz Polynomials of Composite Graphs," Applicable Analysis and Discrete Mathematics, Vol. 2, No. 2, 2008, pp. 285-296. doi:10.2298/AADM0802285E

[23] S. Hossein-Zadeh, A. Hamzeh and A. R. Ashrafi, "WienerType Invariants of Some Graph Operations," Filomat, Vol. 29, No. 3, 2009, pp. 103-113. doi:10.2298/FIL0903103H

[24] M. H. Khalifeh, H. Yousefi-Azari and A. R. Ashrafi, "The First and Second Zagreb Indices of Some Graph Operations," Discrete Applied Mathematics, Vol. 157, No. 4, 2009, pp. 804-811. doi:10.1016/j.dam.2008.06.015

[25] F. Harary, "Graph Theory," Addison-Wesley, Reading, 1969.

[26] N. Trinajstic, "Chemical Graph Theory," CRC Press, Boca Raton, 1992. 